\title{
Evaluación bajo microscopio electrónico de barrido de sellantes de fosas y fisuras hidrófobos e hidrófilos, bajo diferentes condiciones de humedad: Un estudio in vitro
}

\author{
Eyra Elvyra Rangel Padilla ${ }^{1}$; \\ Hector Alejandro Ramírez Peña ${ }^{1}$; \\ Hector Ramón Martínez-Menchaca ${ }^{2}$; \\ Gerardo Rivera Silva ${ }^{3}$; \\ Roberto Valencia Hitte ${ }^{4}$; \\ Maribel Lozano Longoria ; \\ Gustavo Israel Martínez González ; \\ Roberto Espinosa Fernández ${ }^{7}$
}

\section{Resumen}

Objetivo: Evaluar el efecto de diferentes marcas de selladores de fosas y fisuras hidrófobos e hidrófilos en Microscopio electrónico de barrido de emisión de campo (MEB-EC) expuestos a humedad relativa. Materiales y Métodos: Se utilizaron 40 premolares extraídos con fines ortodóncicos, se dividieron al azar en 8 grupos: Grupo 1: sellador Clinpro® (ambiente seco), Grupo 2: sellador Clinpro® (ambiente húmedo), Grupo 3: sellador Embrace WetBond® (ambiente seco), Grupo 4: sellador Embrace WetBond® (ambiente húmedo), Grupo 5: sellador UltraSeal XT Hydro® (ambiente seco), Grupo 6: sellador UltraSeal XT Hydro® (ambiente húmedo), Grupo 7: sellador UltraSeal XT Plus® (ambiente seco) y el Grupo 8 sellador UltraSeal
XT Plus ${ }^{\circledR}$ (ambiente húmedo). Resultados: El Grupo 1 (Clinpro®/ambiente seco) Mostró valores de adhesión al esmalte superiores, de forma estadísticamente significativa, en relación a los demás grupos y mejor calidad en la formación de "tags" en comparación con los otros grupos, independientemente si el ambiente era seco o húmedo. Conclusiones: Los selladores Clinpro $3 \mathrm{M} \AA$, utilizados en condiciones secas o de relativa humedad demostró tener buenos resultados. La resina fluida UltraSeal XT Plus ${ }^{\circledR}$ utilizada en condiciones húmedas no proporcionó buenos resultados observándose poca formación de tags; los selladores hidrófilos (Embrace Wetbond ${ }^{\circledR}$ y UltraSeal XT Hydro $\left.{ }^{\circledR}\right)$ utilizados en un ambiente húmedo tenían una capacidad de adaptación, observándose buena formación de tags; Los selladores hidrófilos usados en am-

\footnotetext{
1. DDS Profesor de Odontología Pediatrica en la Universidad Autónoma de Nuevo León, Monterrey, México.

2. DDS, MSc. Subdirector del departamento de Ortodoncia, Odontología Pediátrica y pacientes Especiales en la Escuela de Odontología de la Universidad de Louisville, Louisville, USA.

3. MD, PhD. Profesor de Morfología y responsable del laboratorio de Ingeniería de tejidos y medicina Regenerativa, Escuela de Medicina, Universidad de Monterrey, Monterrey, México.

4. DDS. Profesor en el Posgrado de Odontología Pediatrica de la Universidad Tecnológica de México, Ciudad de México, México.

${ }^{5}$ DDS. Profesor de la carrera Cirujano Dentista, Universidad de Monterrey, Monterrey, México.

6. DDS, PhD. Profesor de Odontología Pediatrica en la Universidad Autónoma de Nuevo León, Monterrey, México.

7. DDS. Profesor en la Universidad de Guadalajara, Guadalajara, México.
} 
bientes secos no se adaptan a la superficie y tienen una tendencia a fallar en lo que respecta a la adhesión.
Palabras Clave: Formación de tags, selladores hidrofílicos, humedad relativa, microscopio electrónico de barrido.

Artigo Original

\section{Avaliação do microscópio eletrônico de varredura de selantes hidrofóbicos e hidrofílicos sob diferentes condições de umidade: estudo in vitro}

\section{Resumo}

Objetivo: Avaliar o efeito de diferentes marcas de selantes hidrofóbicos e hidrofílicos em microscopia eletrônica de varredura expostos à umidade relativa. Materiais e Métodos: 40 prémolares extraídos para fins ortodônticos foram divididos aleatoriamente em 8 grupos: Grupo 1: Selante Clinpro ${ }^{(a m}$ biente seco), Grupo 2: Selante Clinpro® (ambiente úmido), Grupo 3: Selante WetBond® de Embrace (ambiente seco) Grupo 4: Selante Embrace WetBond ${ }^{\circledR}$ (ambiente úmido), Grupo 5: Selante UltraSeal XT Hydro® (ambiente seco), Grupo 6: Selante UltraSeal XT Hydro® (ambiente úmido), Grupo 7: selante UltraSeal XT Plus (ambiente seco) ) e selante UltraSeal XT Plus® do Grupo 8 (ambiente úmido). Resultados: O Grupo 1 (Clinpro® / ambiente seco) mostrou valores mais elevados de adesão ao esmalte estatisticamente significativo, em relação aos demais grupos. Também apresentou melhor qualidade na formação de "tags" em comparação com os outros grupos, independentemente se o meio estava seco ou úmido. Conclusões: O selante Clinpro $3 \mathrm{M} 囚$, utilizado em condições secas ou de umidade relativa, demonstraram bons resultados. A resina fluida UtraSeal XT Plus ${ }^{\circledR}$, utilizada em condições úmidas, não proporcionou bons resultados com pouca formação de tags. Os selantes hidrofílicos (Embrace Wetbond ${ }^{\circledR}$ e UltraSeal XT Hydro ${ }^{\circledR}$ ), utilizados em um ambiente úmido, tiveram capacidade de adaptação, observando boa formação de tags; Os selantes hidrófilos, utilizados em ambientes se$\cos$, não se adaptam à superfície e tendem a falhar em relação à adesão.

Palavras-chave: Formação de tags, selantes hidrófilos, umidade relativa, microscópio eletrônico de varredura.

Original article

\section{Scanning electron microscope evaluation of hydrophobic and hydrophilic sealants under different humidity conditions: An in vitro study}

\section{Abstract}

Objective: Evaluates the effectiveness of differ- ent brands of hydrophobic and hydrophilic pit and fissure sealants in scanning electron microscopy (SEM) exposed to relative humidity. 
Materials and Methods: 40 premolars extracted for orthodontic purposes were randomly divided into 8 groups: Group 1: Clinpro® sealant (dry environment), Group 2: Clinpro® sealant (wet environment), Group 3: Embrace WetBond ${ }^{\circ}$ sealant (dry environment), Group 4: Embrace WetBond $\AA$ sealant (wet environment), Group 5: UltraSeal XT Hydro® sealant (dry environment), Group 6: UltraSeal XT Hydro® (wet environment) sealant, Group 7: UltraSeal XT Plus sealant ${ }^{\circledR}$ (dry environment) and Group 8 UltraSeal XT Plus® sealant (wet environment). Results: Group 1 (Clinpro® / dry environment) showed higher values of adhesion to the enamel statistically significant, in relation to the other groups. It also showed better quality in the formation of "tags" in com-

\section{Introducción}

Los selladores de fosas y fisuras propuestos por Cueto y Bounocore en los 60s fueron la primera opción de tratamiento de las fisuras para evitar el acumulo de alimentos en las caras oclusales de molares; ${ }^{1}$ estos han evolucionado desde los autopolimerizables a los fotopolimerizables, de selladores transparentes a selladores con color, selladores sin relleno a selladores con relleno; selladores a base de resina a selladores de ionómero de vidrio y la nueva tendencia de selladores hidrófilos (soportan humedad) que tienen la particularidad de permitir la adhesión en condiciones no óptimas, o en situaciones donde los materiales adhesivos no funcionan (humedad).

La humedad relativa dentro de la cavidad oral, el fluido gingival, la sangre, las nano partículas de aceite de la pieza de mano, y la saliva pueden afectar directamente a la adaptación marginal y parison with the other groups, independently if the atmosphere was dry or damp. Conclusions: Clinpro $3 \mathrm{M} \circledast$ sealants, used in dry or relative humid conditions, proved to have good results. The UltraSeal XT Plus ${ }^{\circledR}$ fluid resin used in wet conditions did not provide good results with little tag formation; the hydrophilic sealants (Embrace Wetbond ${ }^{\circledR}$ and UltraSeal XT Hydro $\left.{ }^{\circledR}\right)$ used in a humid environment had a capacity of adaptation, observing good formation of tags; Hydrophilic sealants used in dry environments do not adapt to the surface and have a tendency to fail with regard to adhesion.

Keywords: Tag formation, hydrophilic sealants, relative humidity, scanning electron microscope.

al sellado de cavidades causando varios problemas, entre los cuales se incluyen: microfiltración, fractura marginal, caries secundarias, sensibilidad postoperatoria y decoloración. ${ }^{2}$ Dicho esto, el aislamiento absoluto y control de la contaminación salival son un requisito esencial en todo protocolo de adhesión;, ${ }^{2,3}$ especialmente en molares parcialmente erupcionados o en donde los márgenes de las cavidades se extienden por debajo de los tejidos gingivales o en pacientes con apertura bucal limitada. ${ }^{4,5}$

Los odontólogos no suelen utilizar dique de goma debido a la creencia personal de que son capaces de evitar la contaminación de saliva o líquido dentro de la zona de trabajo6. Además tienen una tendencia a pasar por alto el $95 \%$ o más de humedad relativa presente en la cavidad oral. ${ }^{7}$ Estudios han demostrado que independientemente de la utilización de adhesivos hidrófilos en aislamiento relativo con rollos de 
algodón, la humedad relativa dentro de la cavidad oral como resultado de la respiración del paciente, es suficiente para causar la contaminación, incrementando el riesgo a fallar de los sellantes. ${ }^{8}$ El mismo estudio menciona la justificación del uso de aislamiento absoluto para la colocación de sellantes de fosas y fisuras por ser simple y rápido su uso.

En lo que respecta al efecto de la contaminación salival, en los adhesivos de grabado total, se ha demostrado que disminuye significativamente la fuerza de adhesión. ${ }^{9,10}$ Recientemente se han introducido adhesivos de autograbado con el objetivo principal de reducir el tiempo en los procedimientos de adhesión, aunque también se ha encontrado que la humedad relativa en la cavidad oral y la contaminación de la saliva disminuye la resistencia de la unión y aumenta la microfiltración. ${ }^{10}$ Los nuevos selladores de fosas y fisuras hidrófilos significan un gran paso hacia la prevención dental; lo hacen mediante el establecimiento de nuevos protocolos del uso de selladores que no requieren aislamiento de goma dique; sin embargo, su eficacia no se ha estudiado ampliamente.

\section{Objetivo}

Evaluar la eficacia de diferentes marcas de selladores de fosas y fisuras hidrófobos e hidrófilos en Microscopio electrónico de barrido de emisión de campo (MEB-EC) expuestos a humedad relativa.

\section{Materiales y Métodos}

Se obtuvieron 40 premolares extraídos por razones ortodóncicas los cuales se almacenaron en
Cloramina al 1\% previo a la evaluación. Para el diagnóstico de caries se utilizó la fluorescencia laser (Kavo DiagnoDent, Biberach, Germany) con la finalidad de evaluar que las fisuras estuvieran libres de caries. Los criterios de inclusión incluyen dientes sanos, lo que se estableció mediante el Diagnodent en un rango de 0-15 para incluirse en el estudio; los criterios de exclusión incluyen dientes con selladores previos en los premolares, cavidades visibles, evaluación en el Diagnodent de 15 o más, la presencia de fracturas del esmalte en las fisuras. ${ }^{11}$ Los dientes fueron aleatoriamente divididos en 8 grupos (5 premolares en cada grupo), se establecieron 2 protocolos: 1 ambiente seco que consistió en secar con aire hasta observar deshidratación de la fisura y ambiente húmedo que en el que se procedió a humedecer la fisura previo a la colocación del sellador. (Tabla 1)

Se distribuyeron los grupos de la siguiente manera: Grupo 1 sellador Clinpro® en ambiente seco (3M); Grupo 2 sellador Clinpro® ambiente húmedo; Grupo 3 sellador Embrace Wetbond® ambiente seco; Grupo 4 sellador Embrace Wetbond ${ }^{\circledR}$ ambiente húmedo; Grupo 5 sellador UltraSeal XT Hydro® ambiente seco; Grupo 6 sellador UltraSeal XT Hydro® ambiente húmedo; Grupo 7 sellador UltraSeal XT Plus ${ }^{\circledR}$ ambiente seco; Grupo 8 sellador UltraSeal XT Plus® ambiente húmedo. (Tabla 1)

\section{Colocación del Sellador}

Los dientes fueron limpiados con pieza de mano de baja velocidad y una copa de hule impregnada con una pasta de piedra pómez libre de flúor, después enjuagados con agua; se realizó el protocolo de desproteinización del esmalte con hipoclorito de sodio al $5.25 \%$ por 1 minuto, 


\section{Selladores usados en el estudio.}

- Sellador de fosas y fisuras Clinpro ${ }^{\circledR}(3 \mathrm{M}$ ESPE Dental Products, St, Paul MN, USA) Sellador con $8 \%$ de relleno.

- Sellador de fosas y fisuras hidrófilo Embrace WetBond ${ }^{\circledast}$ (PULPDENT/Gaba, Water-town, MA, USA) sellador con $36.6 \%$ de relleno.

- Sellador de fosas y fisuras UltraSeal XT Plus ${ }^{\circledast}$ (Ultradent Products Inc. USA) Resina fluida con $55 \%$ de relleno.

- Sellador de fosas y fisuras Ultraseal XT Hydro ${ }^{\bullet}$ (Ultradent Products Inc. USA) Resina fluída hidrófila con $55 \%$ de relleno.

\section{Grupos de estudio.}

Grupo 1. Posterior a la desproteinización, se realizó grabado total, se lava con agua y se precipita el agua con jeringa triple, y se seca por 5 segundos con aire a presión para dejar la superfície del esmalte totalmente seca, se coloca el sellador Clinpro directamente en la superficie de la cara occlusal siguiendo las lineas de desarrollo con un explorador \#5, seguido de la aplicación del sellador se fotopolimeriza con una lámpara LED Elipar 2 ( Max, $1200 \mathrm{~mW} / \mathrm{cm} 2$; 3M Dental products, St Paul Mn. USA), posterior a esto se coloca la pieza dental en agua bio-destilada (ddH20).

Grupo 2. El mismo protocolo de grabado total posterior a la desproteinización, se lava por 15 segundos y se seca por 5 segundos con jeringa triple, En el grupo de ambiente húmedo, posterior a la desproteinización, una gota de agua fue aplicada en la cara oclusal por 5 segundos para simular la contaminación, se procedió a eliminar con aire de la jeringa triple durante 5 segundos, posterior a esto se aplico el sellador y se polimerizó con una Lampara de LED Elipar $2^{\circledR}$ (3M Dental products, St Paul Mn. USA), seguido de la fotopolimeración y se almacena en agua bio-destilada (ddH20).

Grupo 3. Se repite el mismo protocolo que el realizado con el grupo 1 usando Embrace Wet-Bond ${ }^{\circledR}$

Grupo 4. Se repite el mismo protocolo que el realizado con el grupo 2 usando Embrace Wet-Bond ${ }^{\circledR}$

Grupo 5. Se repite el mismo protocolo que el realizado con el grupo 1 usando UltraSeal XT HYDRO ${ }^{\circledR}$

Grupo 6. Se repite el mismo protocolo que el realizado con el grupo 2 usando UltraSeal XT HYDRO.

Grupo 7. Se repite el mismo protocolo que el realizado con el grupo 1 usando UltraSeal XT Plus ${ }^{\circledast}$

Grupo 8. Se repite el mismo protocolo que el realizado con el grupo 2 usando UltraSeal XT Plus

Tabla 1. 
posterior a este paso se realizó el grabado ácido por 15 segundos. ${ }^{12}$

Se utilizo humedad relativa del $90 \%$ y una temperatura de $25^{\circ} \mathrm{C}$ centígrados con la finalidad de realizar el tratamiento simulando las condiciones de la cavidad bucal.

En el grupo de ambiente húmedo, posterior a la desproteinización y el grabado ácido, una gota de agua fue aplicada en la cara oclusal por 5 segundos para simular la contaminación, se procedió a eliminar con aire de la jeringa triple durante 5 segundos, posterior a esto se aplico el sellante y se polimerizó con una Lampara de LED Elipar ${ }^{\circledR}(3 \mathrm{M}$ Dental products, St. Paul Mn. USA), esto se aplicó a los dientes correspondientes a este grupo.

En el grupo de ambiente seco, posterior a la desproteinización y grabado ácido se secó la fisura con aire de la jeringa triple y se colocó el sellador correspondiente al grupo estudiado. Los dientes fueron almacenados en agua doble destilada (ddH2O).

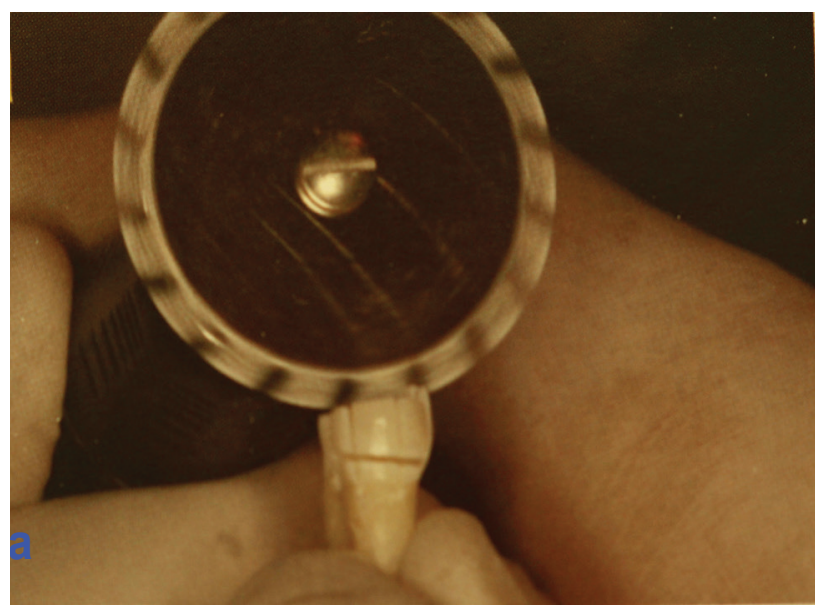

\section{Cortes y preparación para evaluación en el Microscopio electrónico de barrido de emisión de campo.}

Todos los premolares usados en el estudio fueron seccionados buco-lingualmente en tres partes de 5 milímetros, desde la cara oclusal hacia cervical con discos rotatorios de diamante de 1 solo filo Brasseler Diamond Rotating (Brasseler Dental, GA, USA). ${ }^{13}$ Fig. 1 Una vez realizados los cortes se obtuvieron 15 muestras por grupo, resultando un total de 120 muestras, las cuales fueron pulidas con tiras de lija de grano fino hasta el grano 2000; para remover la smear layer (barro dentinario) se utilizó ácido fosfórico al $4 \%$ por 5 segundos en cada porción del diente seccionado. Cada muestra obtenida fue enjuagada con agua destilada (ddH2O) y posteriormente se inició la deshidratación, este último es un procedimiento necesario ya que comúnmente se utilizan medios de inclusión que no son miscibles en agua, en este caso utilizamos soluciones con alcohol a diferentes porcentajes distribuídos de la siguiente manera: Día 1,

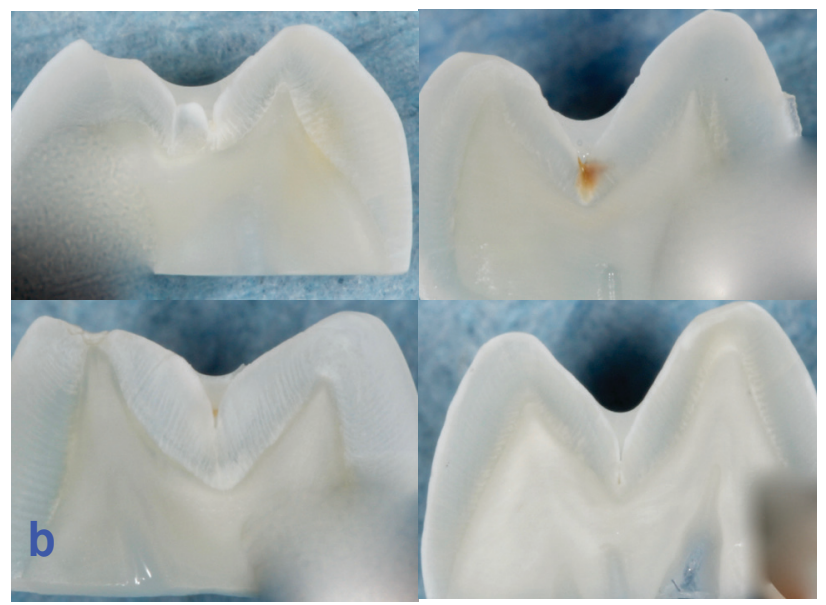

Figura 1. 1a. Cortes con discos rotatorios de diamante de 1 filo (Brasseler Diamond Rotating, Brasseler Dental, GA, USA), 1b. Cortes realizados a las muestras. 

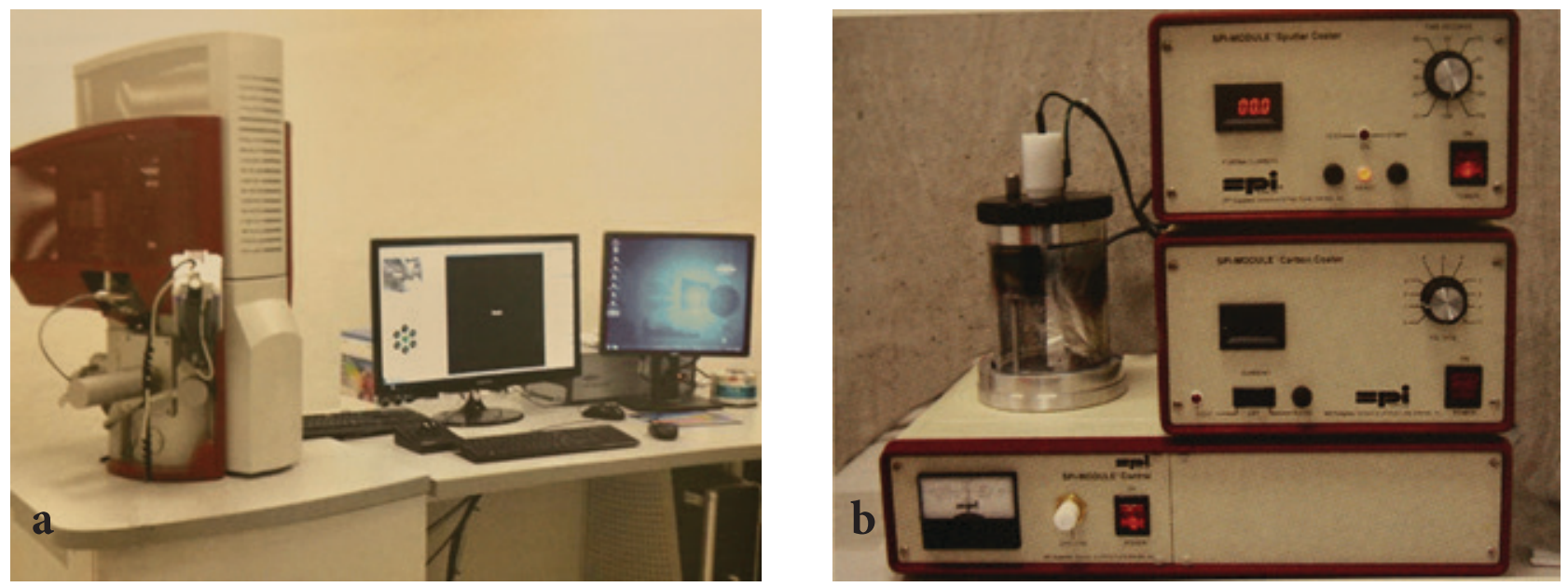

Figura 2. 2a.Microscopio Electrónico de Barrido de Emisión de Campo MEB-EC, (SEM, JEOL JSM 6610 LV, EAG Corporate, CA, USA); 2b. Sputter y recubrimiento de Carbon SPI (Denton Vacuum Desk V., Corporate Hedquaters Denton Vacuum, Morestown, USA). Centro Universitario de Ciencias Exactas e Ingenierías (CUCEI), Guadalajara Jalisco. Foto Cortesías del Libro: Microscopía electronica y su aplicación en la odontología. 1ª. Edicion Guadalajara Jal: AMAT; 2013.

$70 \%$ agua, $30 \%$ alcohol; día 2, 50\% agua: $50 \%$ alcohol; día 3, 30\% agua: 70\% alcohol; día 4, 20\% agua: $80 \%$ alcohol; día 5, 10\% agua: $90 \%$ alcohol y día 7 100\% alcohol. ${ }^{14}$

\section{Evaluación en Microscopio electrónico de barrido de Emisión de Campo (MEB-EC)}

Las muestras fueron recubiertas con materiales conductores ya que las mismas no son conductoras per se, se utilizó la unidad de Sputter y recubrimiento de Carbon SPI (Denton Vacuum Desk V., Corporate Hedquaters Denton Vacuum, Morestown, USA) para impregnación, se utilizó el metal Oro con la finalidad de asegurar la conductividad eléctrica y la emisión de un gran número de electrones secundarios mediante el bombardeo de electrones. ${ }^{15}$ Las muestras fueron evaluadas en el MEB-EC, (SEM, JEOL JSM 6610 LV, EAG Corporate, CA, USA), (Fig. 2) para medir la integridad del sellador se realizó un análisis de formación de tags entre el sellador y el esmalte lo cual se analizó en cada fisura de acuerdo a la propuesto por Celeberti y Lussi (2007), ${ }^{13}$ los cuales propusieron la siguiente clasificación:

Calidad 0. No se encuentran gaps marginales (brechas), existe formación de tags medibles dentro de la fisura, penetración regular presente.

Calidad 1 No se encuentran gaps marginales, existe formación de tags medibles dentro de la fisura con penetración no ideal del material.

Calidad 2: No se encuentran gaps marginales, los bordes del sellador son irregulares, la penetración de la fisura no es medible.

Calidad 3: Se encuentran gaps marginales claramente visibles.

Calidad 4: Ausencia total de áreas selladas. ${ }^{13}$ (Figura3) 


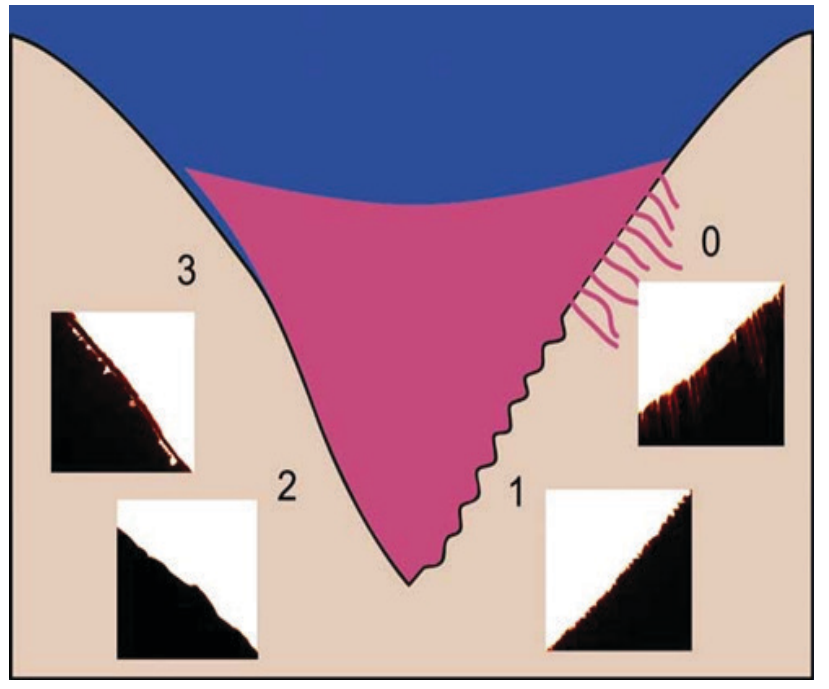

Figura 3. Representación esquemática de la formación de tags. Celiberti-Lussi (2007) $)^{13}$

\section{Análisis Estadístico}

Para el análisis de datos se utilizaron intervalos de confianza OR (odds ratios) en los que se busca el número de muestras con formación de tags y el número de muestras sin formación de tags, esto en las fisuras de los selladores, en los dos ambientes establecidos (ambiente seco y ambiente húmedo). El análisis estadístico utilizado fue el Análisis de varianza (ANOVA) así como el Test de Tukey (HSD) complementario para considerar la significancia estadística. La evaluación de los datos obtenidos se realizó usando el software estadístico (SPSS 23rd versión). Se usaron métodos ajustados para obtener las muestras para el análisis, para este análisis se obtuvo un valor de P menor al .05\% considerado estadísticamente significativo.

\section{Resultados}

Los 40 dientes se dividieron en 8 grupos de 5 dientes cada uno, los cuales fueron distribuidos de la siguiente manera: Grupo 1 sellador
Clinpro® en ambiente seco (3M); Grupo 2 sellador Clinpro® ambiente húmedo; Grupo 3 sellador Embrace Wetbond $₫$ ambiente seco; Grupo 4 sellador Embrace Wetbond ${ }^{\circledR}$ ambiente húmedo; Grupo 5 sellador UltraSeal XT Hydro® ambiente seco; Grupo 6 sellador UltraSeal XT Hydro® ambiente húmedo; Grupo 7 sellador UltraSeal XT Plus ${ }^{\circledR}$ ambiente seco; Grupo 8 sellador UltraSeal XT Plus ${ }^{\circledR}$ ambiente húmedo. Se examinaron un total de 120 muestras $(n=120)$ con una distribución de 15 muestras $(n=15)$ a cada grupo que se evaluó.

Los análisis que fueron obtenidos de cada muestra, nos indicaron que el grupo G1 (Clin-pro ${ }^{\circledR}$ en ambiente seco) demostró una excelente capacidad de adhesión a la superficie del esmalte en la fisura ya que se observó una extensa formación de tags en la fisura, (Fig. 4 A 1-4). El grupo G2 (Clinpro® en ambiente húmedo) de selladores en "ambiente húmedo" demostró tener una formación de tags medibles. (Fig. 4 B 1-4) Para el grupo G3 (Embrace Wetbond $\AA$ en ambiente seco) mostró problemas en la adhesión, en el sellador se mostraron fracturas, y en algunas zonas se localizaron gaps en la unión entre el sellador y el esmalte, incluso se evidenció el desprendimiento del esmalte hacia la restauración (Fig. 4 C 1-4). En el Grupo G4 (Embrace Wetbond ${ }^{\circledR}$ en estado húmedo) se observó mejor penetración del sellador en el esmalte, ya que se observaron tags medibles dentro de la fisura, sin embargo, fracturas notables fueron evidentes entre el esmalte y el segmento adherido (Fig. 4 D 1-4); El grupo G5 (UltraSeal XT Hydro® en ambiente seco) reveló menor desprendimiento en la interfase entre el sellador y el esmalte de la fisura (Fig. 5 A 1-4); El grupo G6 (UltraSeal XT Hydro® ambiente húmedo), reveló muy buena adhesión en la interface formada por el sellador y esmalte, y se observó una formación de tags 


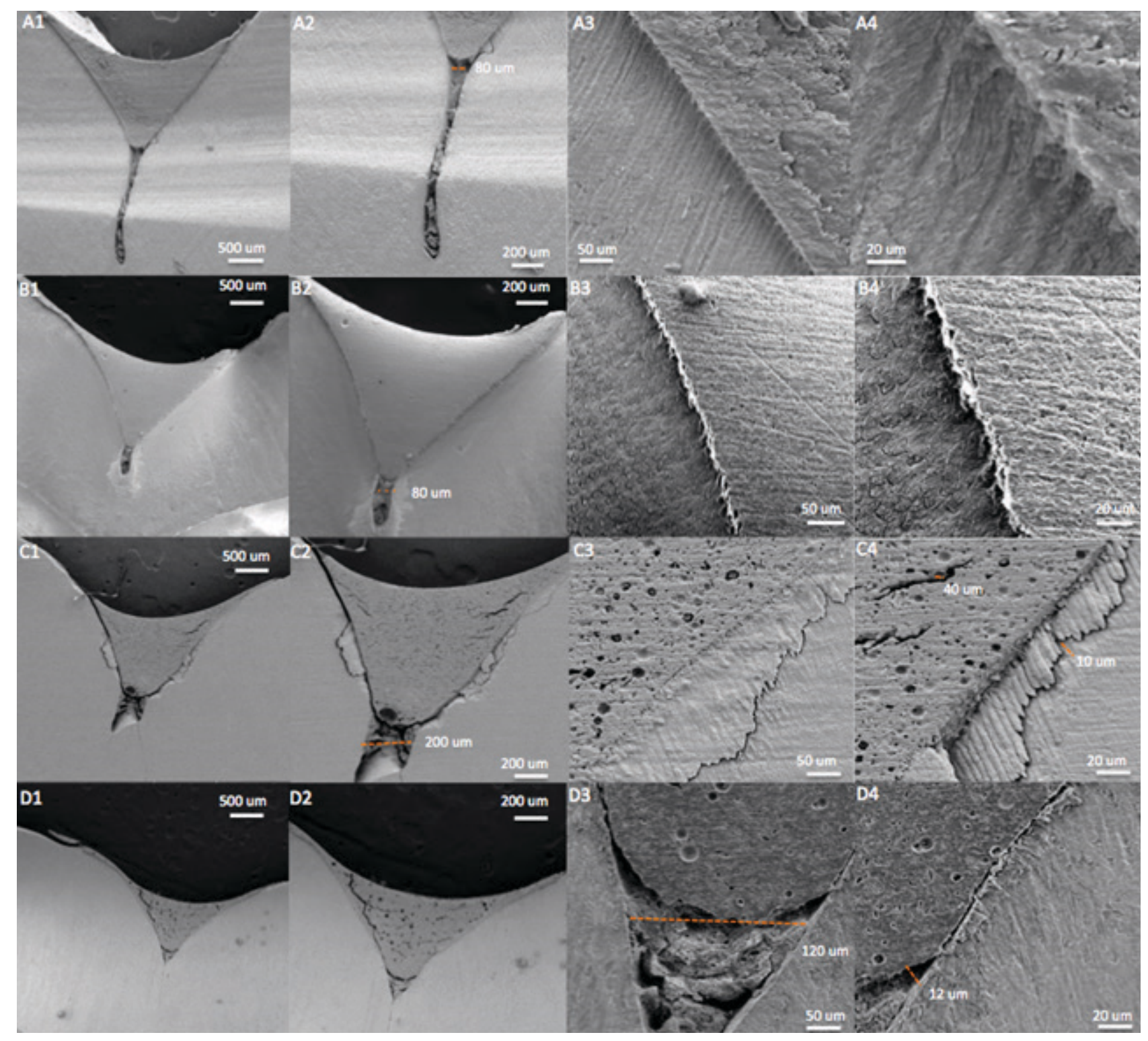

Figura 4. Imágenes del Microscopio electrónico de barrido que muestran el efecto de los selladores Clinpro® y Embrace WetBond ${ }^{(1-4 ~ 500, ~ 200, ~ 50, ~} 20$ um).

A.-Se observa un sellado perfecto con Clinpro® ambiente seco (A 1-4). B.- Se observa una buena formación tags en toda el contorno del sellador Clinpro® en ambiente húmedo. (B 1-4). C.- Se observa una adaptación muy deficiente y se observan fracturas en la interface esmalte-sellador del sellador Embrace WetBond® en ambiente seco (C 1-4). D.- Se observa buena adaptación del sellador con un mínimo de fracturas del sellador Embrace WetBond® en ambiente húmedo (D 1-4).

de muy buena calidad con penetración ideal (Fig. 5 B 1-4); G7 (UltraSeal XT Plus® en ambiente seco), presentó formación de tags en la interfase del sellador y el esmalte, sin evidencia de desprendimiento del material (Fig. 5 C 1-4); en el G8 (UltraSeal XT Plus® ambiente húmedo) se observó nula adhesión con el esmalte de la fisura; se observaron gaps (brechas) en los márgenes de las restauración sin formación de tags de resina (Fig. 5 D 1-4). 


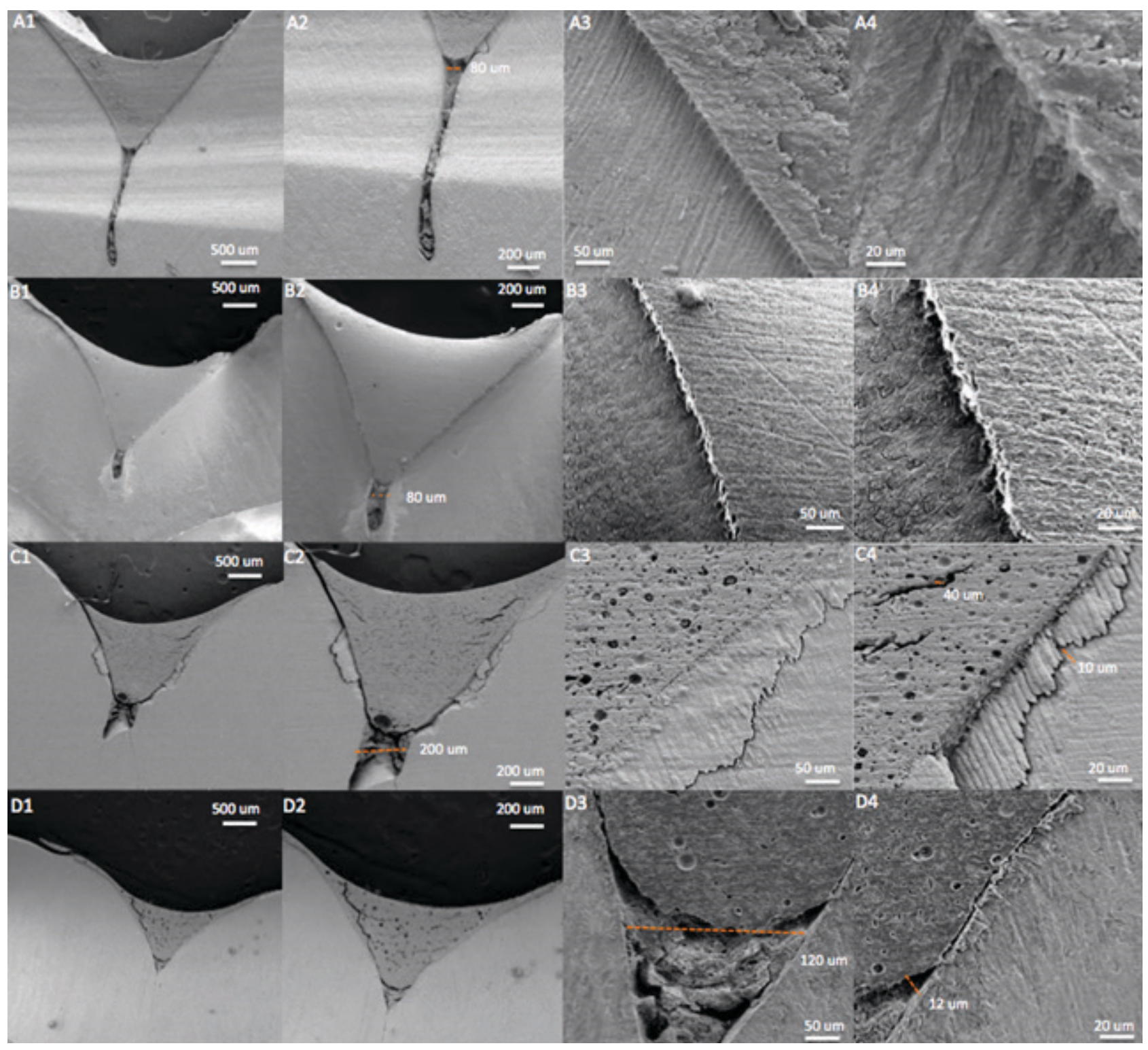

Figura 5. Imágenes obtenidas del Microscopio electrónico de barrido de emisión de campo para observar el sellador UltraSeal XT hidro® y UltraSeal XT plus®. (fotos 1-4 a 500, 200, 50 y 20 um).

A.- Mínimo desprendimiento y pobre penetración después de la aplicación del sellador UltraSeal Xt hidro® en ambiente seco (A 1-4). B.- Se observan gaps en el borde de la in-terface Sellador-esmalte, menos fracturas y poca formación de tags usando UltraSeal XT hidro ${ }^{\circledR}$ en ambiente húmedo (B 1-4). C.- Se observa una perfecta union entre el sellador y el esmalte del UltraSeal $X T$ plus ${ }^{\circledR}$ en ambiente seco (C 1-4). D.- Se observa formación completas de gaps en toda la unión del sellador con el esmalte con el sellador UltraSeal XT plus® en ambiente húmedo. (D 1-4).

Los resultados obtenidos, fueron evaluados estadísticamente mediante el análisis de la varianza (ANOVA), observándose diferencias significativas entre los grupos ( $\mathrm{p}=0-001)$ (gráfica 1, tabla 2 y 3); La utilización de la prueba de comparación múltiple de superficies HSD de Tukey se realizó con la finalidad de comparar entre los diferentes grupos de selladores. Estos datos indican 


\begin{tabular}{|c|c|c|c|c|c|c|c|c|c|c|c|c|}
\hline \multicolumn{13}{|c|}{ Formación de tags en los diferentes tipos de selladores. } \\
\hline & \multicolumn{2}{|c|}{ 0 } & \multicolumn{2}{|c|}{1} & \multicolumn{2}{|c|}{2} & \multicolumn{2}{|c|}{3} & \multicolumn{2}{|c|}{4} & \multicolumn{2}{|c|}{ Total } \\
\hline & $\mathbf{n}$ & $\%$ & $\mathbf{n}$ & $\%$ & $\mathbf{n}$ & $\%$ & $\mathbf{n}$ & $\%$ & $\mathbf{n}$ & $\%$ & $\mathbf{n}$ & $\%$ \\
\hline Clinpro $^{\circledR}$ seco & 12 & 92.31 & 1 & 7.69 & 0 & 0.00 & 0 & 0.00 & 0 & 0.00 & 13 & 100 \\
\hline $\begin{array}{l}\text { Clinpro }^{\circ} \\
\text { húmedo }\end{array}$ & 9 & 81.82 & 2 & 18.18 & 0 & 0.00 & 0 & 0.00 & 0 & 0.00 & 11 & 100 \\
\hline $\begin{array}{l}\text { Embrace } \\
\text { Wetbond }^{\oplus} \\
\text { seco }\end{array}$ & 4 & 33.33 & 5 & 41.67 & 2 & 16.67 & 1 & 8.33 & 0 & 0.00 & 12 & 100 \\
\hline $\begin{array}{l}\text { Embrace } \\
\text { Wetbond }^{\circledR} \\
\text { húmedo }\end{array}$ & 0 & 0.00 & 0 & 0.00 & 1 & 8.33 & 9 & 75.00 & 2 & 16.67 & 12 & 100 \\
\hline $\begin{array}{l}\text { Ultra Seal } \\
\text { Hydro }^{\circledR} \text { seco }\end{array}$ & 4 & 33.33 & 8 & 66.67 & 0 & 0.00 & 0 & 0.00 & 0 & 0.00 & 12 & 100 \\
\hline $\begin{array}{l}\text { Ultra Seal } \\
\text { Hydro } \\
\text { húmedo }\end{array}$ & 8 & 66.67 & 2 & 16.67 & 2 & 16.67 & 0 & 0.00 & 0 & 0.00 & 12 & 100 \\
\hline $\begin{array}{l}\text { Ultra Seal XT } \\
\text { Plus }^{\oplus} \text { seco }\end{array}$ & 12 & 100 & 0 & 0.00 & 0 & 0.00 & 0 & 0.00 & 0 & 0.00 & 12 & 100 \\
\hline $\begin{array}{l}\text { Ultra Seal XT } \\
\text { Plus }{ }^{\circledR} \text { húmedo }\end{array}$ & 0 & 0.00 & 0 & 0.00 & 0 & 0.00 & 5 & 38.46 & 8 & 61.54 & 13 & 100 \\
\hline $\begin{array}{l}X 2=145.39 \\
p=0.0001\end{array}$ & & & & & & & & & & & & \\
\hline
\end{tabular}

Tabla 2.

que los selladores en ambiente seco el grupo G4 (Embrace Wetbond ${ }^{\circledR}$ en ambiente húmedo) y el grupo G8 (UltraSeal Plus® en ambiente húmedo) difieren significativamente de los otros grupos evaluados, $(\mathrm{p}=0.0001)$, (Tabla 4$)$

\section{Discusión.}

Se evaluó el uso de selladores de fosas y fisuras como prevención de caries dental que continúa teniendo un lugar significativo en la odontología contemporánea. Rivas (2002) ${ }^{17}$ definió los selladores de fosas y fisuras, como una resina que se aplica y retiene micro-mecánicamente a la super- ficie del esmalte. Así se consigue el sellado y el aislamiento, protegiendo la estructura anatómica del diente del medio ambiente. Este tratamiento se realiza principalmente en las superficies oclusales como un procedimiento eficaz y preventivo de la caries dental.

En el presente estudio realizado in vitro, se evaluó la adaptación marginal y la unión al esmalte de los selladores de fosas y fisuras en premolares, en dos tipos de situaciones comunes en la práctica clínica de rutina: Ambiente seco y ambiente húmedo, este último para simular un ambiente contaminado con saliva. Las observaciones se realizaron utilizando microscopio electrónico de barrido de emisión de 


\begin{tabular}{|c|c|c|c|c|c|}
\hline \multicolumn{7}{|c|}{ Análisis de varianza (ANOVA) } \\
\hline & Sum of squares & df & Means squares & F-Ratio & Sig. \\
\hline Entre grupos & 173.141 & 7 & 24.734 & 78.008 & .000 \\
\hline $\begin{array}{c}\text { Within groups } \\
\text { or error }\end{array}$ & 28.220 & 89 & .317 & & \\
\hline Total & 201.361 & 96 & & & \\
\hline
\end{tabular}

Tabla 3.

campo (MEB-EC). Aunque es un estudio in vitro, se trató de imitar las condiciones in vivo, mediante el uso de humedad relativa previo a la colocación de los selladores, ya que hacer que las condiciones del estudio in vitro se asemejen a las del estudio in vivo, es complicado, uno de los principales problemas fue el de proveer ciclos térmicos a los dientes en estado in vitro, con la finalidad de que los cambios de temperatura tuvieran alguna influencia significativa en la interface esmalte-sellador en este estudio, de lo cual no se obtuvieron resultados medibles que tuvieran significancia hacia los cambios de temperatura y la correspondiente repercusión en las restauraciones. Gracias a esto se corroboró la limitada posibilidad de imitar el estado in vivo, sin embargo los problemas de formación de tags o gaps analizados en MEB-EC no tendrían relevancia al momento de la evaluación de la adhesión de los selladores al esmalte; otra variable a evaluar es la microfiltración en la interface entre esmalte y sellador, como consecuencia de la fotopolimeración sin embargo estos datos no han sido significativos. Independientemente de que la morfología de la fisura tenga un papel en la penetración del sellador, lo mas importante puede radicar en el tratamiento previo de las fisuras. La desproteinización es un tratamiento necesario para la remoción del contenido orgánico dentro de la fisura; Valencia y cols. (2014) ${ }^{16}$ concluyen que la desproteinización de la estructura dental previo al grabado ácido proporcionaba una mejor adaptación del material sellador en la estructura dental, ${ }^{16}$ Por lo tanto la desproteinización produce una pérdida menor de material sellante a través del tiempo. ${ }^{12}$ En este proyecto, el protocolo de desproteinización del esmalte obtuvo buenos resultados en las muestras analizadas, aunque otros estudios han realizado la preparación previa al grabado ácido con diferentes técnicas, (Er: YAG-Aire abrasivo), no encuentran diferencias significativas entre los selladores y las fisuras tratadas,20 sin embargo Espinosa y Cols. $(2007)^{16}$ indican que al usar hipoclorito de sodio ( $\mathrm{NaOCl}$ al $5.25 \%$ ) se incrementa la superficie retentiva en un $45 \%$.

Aunque en este estudio se estableció el uso de la desproteinización, en algunas muestras obtenidas, la anatomía de la fisura era tipo Y2, la cual se caracteriza por tener constricción al fondo de la fisura, lo que impide la remoción completa de materiales orgánicos, contrasta con el hecho de que se ha demostrado que aislar los microorganismos dentro de la fisura evita el desarrollo de lesiones cariosas mas profundas; por lo que un sellador colocado en una de estas fisuras podría ser exitoso de acuerdo a lo dicho por Celiberty y Lussi (2005) $)^{13}$ en el que el sellador herméticamente adherido al esmalte puede cortar el sustrato para los microorganismos atrapados en la fisura y la recolonización de la fisura. 
La evolución de los selladores tolerantes a la humedad se ha convertido en la justificación más importante para dejar de usar dique de hule, Knight y cols, (1994) ${ }^{18}$ evaluaron la adaptación $\mathrm{y}$ una filtración marginal de restauraciones en esmalte realizadas con aislamiento relativo con rollos de algodón, en donde concluyen que hubo filtración marginal mayor en los casos en los que se usó aislamiento relativo. ${ }^{18}$

En las restauraciones realizadas con aislamiento absoluto (dique de hule) la investigación co-

\section{Prueba HSD de Tukey de comparaciones mútiples de las evaluaciones entre selladores con diferencias estadísticamente significativas}

\begin{tabular}{|c|c|c|c|}
\hline \multicolumn{2}{|c|}{ Sellador } & Diferencia de medias & Valor $\mathrm{p}$ \\
\hline \multirow{3}{*}{ Clinpro seco } & Embrace Wetbond seco & $-3,538$ & .000 \\
\hline & Ultra seal Hydro húmedo & $-3,006$ & .000 \\
\hline & Ultra seal Hydro seco &,$- 923^{*}$ & .002 \\
\hline \multirow{2}{*}{ Clinpro húmedo } & Embrace Wetbond seco & $-3,434^{*}$ & .000 \\
\hline & Ultra seal Hydro húmedo & $-2,902^{*}$ & .000 \\
\hline \multirow{3}{*}{$\begin{array}{l}\text { Embrace Wet-bond } \\
\text { húmedo }\end{array}$} & Embrace Wetbond seco & $-3,615^{\star}$ & .000 \\
\hline & Ultra seal Hydro húmedo & $-3,083^{*}$ & .000 \\
\hline & Ultra seal Hydro seco & $-1,000^{*}$ & .001 \\
\hline \multirow{6}{*}{ Embrace Wet-bond seco } & Clinpro seco & $3,538^{*}$ & .000 \\
\hline & Clinpro vapor & $3,434^{*}$ & .000 \\
\hline & Embrace Wetbond húmedo & $3,615^{*}$ & .000 \\
\hline & Ultra seal Hydro seco & $2,615^{*}$ & .000 \\
\hline & Ultra seal XT plus seco & $3,115^{\star}$ & .000 \\
\hline & Ultra seal XT plus vapor & $2,949^{*}$ & .000 \\
\hline \multirow{6}{*}{ UltraSeal Hydro húmedo } & Clinpro seco & $3,006^{*}$ & .000 \\
\hline & Clinpro vapor & $2,902^{*}$ & .000 \\
\hline & Embrace Wetbond húmedo & $3,083^{\star}$ & .000 \\
\hline & Ultra seal Hydro seco & $2,083^{\star}$ & .000 \\
\hline & Ultra seal XT plus seco & $2,583^{*}$ & .000 \\
\hline & Ultra seal XT plus vapor & $2,417^{*}$ & .000 \\
\hline \multirow{5}{*}{ Ultra seal Hydro seco } & Clinpro seco & ,923* & .002 \\
\hline & Clinpro vapor &, $818^{\star}$ & .017 \\
\hline & Embrace Wetbond húmedo & $1,000^{*}$ & .001 \\
\hline & Embrace Wetbond seco & $-2,615^{*}$ & .000 \\
\hline & Ultra seal Hydro húmedo & $-2,083^{*}$ & .000 \\
\hline \multirow{2}{*}{ Ultra seal XT plus seco } & Embrace Wetbond seco & $-3,115^{*}$ & .000 \\
\hline & Ultra seal Hydro húmedo & $-2,583^{*}$ & .000 \\
\hline \multirow{2}{*}{ Ultra seal XT plus húmedo } & Embrace Wetbond seco & $-2,949^{*}$ & .000 \\
\hline & Ultra seal Hydro húmedo & $-2,417^{\star}$ & .000 \\
\hline
\end{tabular}

Tabla 4. 


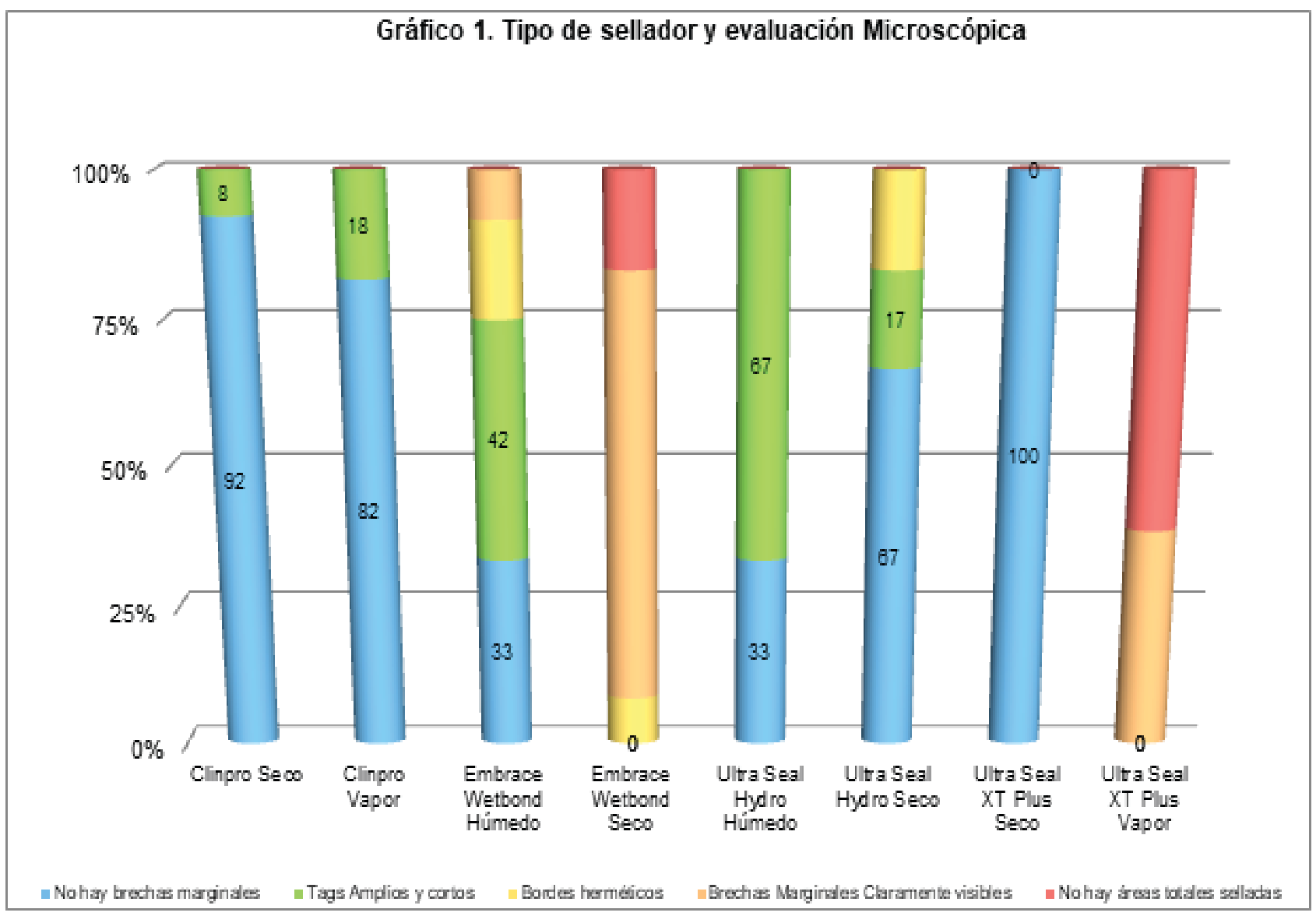

Gráfica 1. Tipo de sellador y evaluación microscópica.

Calidad 0. No se encuentran gaps marginales (brechas), existe formación de tags medibles dentro de la fisura, penetración regular presente.

Calidad 1. No se encuentran gaps marginales, existe formación de tags medibles dentro de la fisura con penetración no ideal del material.

Calidad 2. No se encuentran gaps marginales, los bordes del sellador son irregulares, la penetra-ción de la fisura no es medible.

Calidad 3. Se encuentran gaps marginales claramente visibles.

Calidad 4. Ausencia total de áreas selladas. ${ }^{13}$

rrobora los resultados relacionados a los efectos causados en un ambiente húmedo, en el sentido de que los selladores bajo esta condición mostraron áreas no selladas como en el caso de las muestras del Grupo 8 (UltraSeal XT Plus ${ }^{\circledR}$ colocado en ambiente húmedo) además de la presencia de áreas con brechas marginales (gaps). Sin embargo las muestras del grupo 2
(Clinpro® en ambiente húmedo) demostraron tener buena formación de tags y una integridad adecuada con calidad de tags que oscila entre los rangos de calidad 0 y 1 ; lo que coincide con los estudios previos hechos por Ashwin y Arathi $(2007)^{21}$ en los que se evidenció un buen comportamiento de este sellante en ambiente húmedo. 
En general los 2 grupos de sellantes hidrófobos (Clinpro y UltraSeal XT Plus ${ }^{\circledR}$ ) en “ambientes Secos" muestran excelentes valores adhesivos al esmalte de la fisura, al presentar formaciones de tags medibles (92\% Clinpro®, 100\% UltraSeal XT Plus $\left.{ }^{\circledR}\right)$. Se cree que la ventaja de Clinpro® sobre los otros selladores radica en el bajo porcentaje de relleno.

Los sellantes hidrófilos (Embrace WetBond®y UltraSeal XT Hydro®) presentaron resultados inconsistentes, en el caso del grupo 3 (Embrace WetBond $($ ) en ambiente seco, se observó menos formación de gaps y menos valores positivos (Calidad 3 en el 75\% y calidad 4 17\% de formación de tags), esto difiere al estudio realizado por Khogli et al (2013) el cual mostraba muy buenos resultados de adhesion en ambientes secos y justificaban que posiblemente se quedaban restos de agua, los cuales activaban el sellador Hidrofilico, sin embargo en este estudio se encontró incluso desprendimiento del esmalte hacia la restauración (factor $\mathrm{C}$ ) por la falta de humedad; en el caso del grupo 5 (UltraSeal Xt Hydro®) en ambiente seco se observaron menos complicaciones en las variables estudiadas (Calidad 1:33\%, y calidad 0: 67\%).

Los selladores Clinpro® evaluados en los dos tipos de ambientes (seco y húmedo) se comportaron casi iguales, no hubo diferencia significativa en la formación de tags (92-82\% respectivamente según la clasificación de Celiberti y Lussi) Grafica 1, figura 3; Sin embargo el grupo 7 y 8 (UltraSeal XT Plus®) en ambiente húmedo vs seco, el grupo en ambiente húmedo mostró los peores resultados debido a la contaminación por agua simulando saliva, estos datos corroboran los realizados por Panigrahi et al (2015) en el que concluyen que la saliva o contaminación con humedad afecta la micorfiltración y la adhesión. ${ }^{23}$
El sellador Embrace WetBond $\AA$ obtuvo buenos resultados cuando la superficie del esmalte estaba en condiciones de humedad, estos resultados están en concordancia con los obtenidos en el estudio de Panigrahi et al (2015), en el que encontraron que Embrace Wetbond® podía adherirse en presencia de humedad sin afectar la microfiltración. ${ }^{23}$

En los resultados mostrados por UltraSeal Xt Hydro ${ }^{\circledR}$, también se encontraron mejores resultados en un ambiente húmedo que en seco, basándose en una mejor penetración y adaptabilidad debido a que son hidrófilos. Los selladores sin relleno (Clinpro®) tienen buenos resultados en cuanto a adhesión y formación de tags en la superficie de la fisura. Estudios anteriores realizados por Celiberti, Lussi (2005) y Gunjal y cols (2012) han mostrado resultados similares a los estudiados en técnicas no invasivas (sin ameloplastía). ${ }^{13,25}$ Otro estudio realizado por Kane y cols (2009) comparó Clinpro ${ }^{\circledR}$ vs Embrace ${ }^{\circledR}$ y obtuvo mejores resultados Clinpro contra Embrace ${ }^{\circledR}$, en lo que respecta a la adaptación marginal $^{26}$. Sin embargo, en este estudio se encontró que Clinpro ${ }^{\circledR}$ obtienen excelentes resultados tanto en ambientes húmedos como secos; Mientras que los selladores Embrace WetBond ${ }^{\circledR}$ obtuvieron buenos resultados en ambientes húmedos pero no en secos. Los resultados se explican en sentido a los efectos causados por la contaminación con respecto al proceso de adhesión. ${ }^{22}$

\section{Conclusiones}

A pesar de hay países en los que se pretende que la población de profesionales que colocan selladores de fosas y fisuras se expanda a técnicos de la profesión y profesionales o especialistas de otras áreas de la salud, la conciencia de 
lo sensible de la técnica y el conocimiento del Por otra parte, cuando se justifica el uso de sematerial dental es importante para lograr una excelente adaptación marginal al utilizarlos. Los lladores hidrófilos en cuanto a su adaptabilidad selladores Clinpro®, cuando se usan en condiciones relativamente húmedas y secas, proporcionan buenos resultados. El sellador UltraSeal XT Plus ${ }^{\circledR}$ al usarse en condiciones relativamente contaminadas, proporcionan malos resultados en cuanto a la formación de tags y la adhesión. en un ambiente húmedo, Embrace Wetbond $₫$ y UltraSeal XT Hydro ${ }^{\circledR}$ son una buena elección, presentando mejores resultados el UltraSeal XT Hydro®. El sellador hidrófilo Embrace Wetbond $\AA$, cuando se usa en ambiente seco, no se adapta a la superficie y puede presentar problemas que comprometan la adhesión.

\section{Referencias bibliográficas}

1. Cueto EI, Buonocore MG. Sealing of pits and fissures with an adhesive resin: its use in caries prevention. J Am Dent Assoc 1967; 75 (1): 121-8.

2. Espinosa R, Valencia R, Ramirez A, Rangel EE. Efecto en la adhesión al esmalte por contaminación por humedad y saliva; Estudio al MEB-EC. Revista RODYB; 2015; IV (2): 39-43.

3. Yoo Hm, Oh TS, Pererira PN. Effect of saliva contamination on the microshear bond strength of one-step self -etching adhesive systems to dentin. Oper Dent 2006; 31(1): 127-34.

4. Mojon P, Kaltio R, Feduik D, Hawbolt EB, MacEntee MI. Short term contamination of luting cements by water and saliva. Dent Mater 1996; 12(2): 83-7.

5. Berh M, Rosentritt M, Loher H, et al. Changes of cement properties caused by mixing errors: the therapeutic range of different cement types. Dent Mater 2008; 24(9): 1187-93.

6. Park JW, Lee KC. The influence of salivary contamination on shear bond strength of dentin adhesive systems . Oper Dent. 2004; 29(4): 437-42.

7. Gilbert G H, Craig W. Amundson CW, Gordan V, DPBRN Collaborative Group. Rubber dam use during routine operative dentistry procedures: findings from The Dental PBRN. Oper Dent. 2010; 35(5): 491-99.

8. Barghi N, Knight GT, Berry TG. Comparing two methods of moisture control in bonding to enamel: a clinical study. Oper Dent. 1991; 16(4): 130-5.

9. Kameyama A, Asami M, Noro A, Abo H, Hirai Y, Tsunoda M. The effects of three dry-field techniques on intraoral temperature and relative humidity. J Am Dent Assoc. 2011; 142(3): 274-80.

10. Aboushelib MN. Clinical performance of self-etching adhesives with saliva contamination. J Adhes Dent 2011; 13(5): 489-493. 11. Jenson L, Budenz AW, Featherstone J, Ramos-Gomez F, Spolsky VW, Young DA. Clinical protocols for Caries Management by Risk Assessment. J Calif Dent Assoc. 2007; 35 (10): 714-23.

12. Espinosa R, Valencia R, Uribe M, Ceja I, Saadia M. Enamel deproteinization and its effect on acid etching: an in vitro study. J Clin Pediatr Dent. 2008; 33(1): 13-19.

13. Celiberti, P, Lussi A. Use of self-etching adhesive on previously etched intact enamel and its effect on sealant microleakage and tag formation. J Dent. 2005; 33(2): 163-71.

14. Espinosa R, Valencia R, Ceja I. Microscopía electronica y su aplicación en la odonto-logía. 1a. Edicion Guadalajara Jal: AMAT; 2013. 
15. Ceja I, Espinosa R. capítulo 2 en: Microscopía electronica y su aplicación en la odontología. 1a. Edición Guadalajara Jal: AMAT; 2013.

16. Valencia R, Espinosa R, Ruiz G, Ceja I. Metodología para la evaluación de selladores de fosetas y fisuras in vivo, y su comparación con la desproteinizacion del esmalte. RODYB 2014; 3(2): 33-42.

17. Rivas J. Devenir histórico de los selladores de fosetas y fisuras. Revista ADM 2002; LIX (3): 110-113.

18. Knight GT, Berry TG, Barghi N, Burns TR. Effects of two methods of moisture control on marginal microleakage between resin composite and etched enamel: a clinical study. Int J Prosthodont. 1993: 6(5): 475-9.

19. Espinosa R., Valencia R., Ramírez A., Rangel E.E. Efecto en la adhesión al esmalte por contaminación por humedad y saliva; Estudio al MEB-EC. RODYB. 2015; 4(2): 39-43.

20. Ciucchi P, Neuhaus KW, Emerich M, Peutzfeldt A, Lussi A. Evaluation of different types of enamel conditioning before application of a fissure sealant. Lasers Med Sci. 2015; 30(1): 1-9.

21. Ashwin R, Arathi R. Comparative evaluation for microleakage between Fuji-VII glass ionomer cement and light-cured unfilled resin: A combined in vivo in vitro study. J Indian Soc Pedod Prev Dent 2007; 25: 86-7.

22. Al-Jobair A. Scanning electron microscope analysis of sealant penetration and adaptation in contaminated fissures. J Indian Soc Pedod Prev Dent 2013; 31(3): 169-74.

23. Panigrahi A, Srilatha KT, Panigrahi RG, Mohanty S, Bhuyan SK, Bardhan D. Bond Strength of Embrace Wetbond Hydrophilic Sealant in Different Moisture Contamina-tion: An In-Vitro Study. J Clin Diagn Res 2015; 9(7): ZC23-ZC25.

24. Khogli AE, Cauwels R., Vercruysse C., Verbeeck R., Martens l. Microleakage and penetration of a hydrophilic sealant and a conventional resin based sealant as a function of preparation techniques: a laboratory study. Int J Paediatric Dent. 2013 (1); 23 : $13-22$.

25. Gunjal S, Nagesh L, Raju HG. Comparative evaluation of marginal integrity of glass ionomer and resin based fisure sealants using invasive and non-invasive techniques. An in vitro Study. Indian Dent Res 2012; 23(3): 320-325.

26. Kane B., Karren J., Garcia-Godoy C., Garcia-Godoy F. Sealant adaptation and pene-tration into occlusal fissures. Am J Dent. 2009; 22(2): 89-91.

27. Güçlü ZA, Dönmez N, Hurt A. Coleman N. Characterisation and microleakage of a new hydrophilic fissure sealantUltraSeal Xt Hydro. J Appl Oral Sci. 2016; 24(4): 344-51.

Recibido: $16 / 04 / 2017$

Aceptado: 18/09/2017

Correspondencia: Héctor Alejandro Ramírez. odontologiapediatrica@gmail.com.

Av. Leones 145 L 4, Cumbres Elite, Monterrey, Nuevo León, México. Tel. 5218114777666. 